\title{
Vehicular Communication for Internet-of-Things
}

\author{
Weiqi Xiang ${ }^{1, a}$, and Mao Wang ${ }^{2}$ \\ ${ }^{1}$ Nanjing University of Science and Technology, Nanjing 210094, China \\ ${ }^{2}$ Southeast University, Nanjing 211189, China \\ axiangweiqi@njust.edu.cn
}

Keywords: IoT; LTE-V; 5G; vehicular communication

\begin{abstract}
Since the emergence of the Internet of Things (IoT), a larger number of terminal devices have joined the wireless network, making the wireless network environment more complex and making the date volume explosive growth. The huge data traffic may be required that the max end-to-end latency is as low as 3ms and the reliability is as high as 99.999\%. Existing communication network cannot meet these requirements. This paper analyzes the limitations of existing vehicular communication technologies, provides the overview of vehicular communication based the long-term evolution (LTE), and proposes a feasibility to meet the latency requirement to support the IoT of vehicles in $5 \mathrm{G}$.
\end{abstract}

\section{Introduction}

The emergence of automobiles has promoted the development of the economy, the development of economy has further promoted the growth of automobiles, with traffic accidents, traffic congestion and traffic pollution. This has led to the development of integrated transportation management systems in various countries, and has led to the emergence of Intelligent Transportation System (ITS). ITS integrates information technology, digital communication technology, electronic sensing technology, control technology and computer technology, including acquisition systems (such as GPS positioning, infrared radar, etc.), analysis systems (such as information servers, manual decision-making, etc.) and publishing system (such as Internet, mobile phones, car terminals, roadside unit, etc.). At the same time, Internet of Things (IoT) is known as the third wave of the development of the world information industry after the computer and the Internet. IoT is a huge network that refers to real-time collection of any objects or processed that need to be monitored, connected and interacted through various information sensing devices. The vehicular ITS can achieve both the goal of ITS and the access of the vehicle to the IoT network.

The vehicular IoT enables the vehicle to be connected to all moving things, called vehicle to everything (V2X). V2X enables the connected vehicle communicates with not only the service server or internet network (V2N), but also with the nearby infrastructure (V2I), also with the proximity vehicle (V2V), with the closing pedestrians (V2P) [1]. Vehicle IoT puts users in a involute environment, making security be a key for the it. The cellular network has been operating safely, the vehicular IoT can be based on it. [2] has reviewed the current research challenges and opportunities related to the development of secure and safe ITS applications, [3] gives standardization approaches for security vehicle IoT. And vehicle must quickly discovery other user 
equipment (UE), the vehicular IoT can be able to master traffic condition and avoid traffic accident [4] [5].

IoT integrates RFID, infrared sensors, global positioning systems, and wireless communication systems to get traffic information, the IoT Cloud System processes these big dates and sends the results of the analysis to UEs[6][7]. These require a low-latency, highly reliable wireless communication system to support, especially in some scenarios, such as advanced driving, that need to support 3ms, traditional cellular communication consider system throughput to be the most important performance metric [8]. And latency requirement and reliability requirement is indeed beyond what existing mobile communication system can offer.

There has been a goal that significant research efforts have been devoted to V2V communications using IEEE 802.11p-based DSRC technology and ITS G5 standards. 3rd Generation Partnership Project (3GPP) triggered the V2X work and added the V2X application which supports V2X communication using LTE sidelink communication (referred as to LTE-V, LTE-V2X or Cellular V2X). Although DSRC has the advantage of first-mover and its technology tends to mature. LTE-V2X is based on the LTE, and has many late-development advantages. LTEV2X uses more reference symbols to estimate channel more accurately at high speed, has efficient resource selection to decreases conflict, and realize long and short distance transmission using Uu interface and PC5 interface. These advantages tell us that tells us that LTE-V2X has brighter applications prospects, and LTE-V2X can smoothly evolve to 5G, from the perspective of longterm development of intelligent transportation.

This article focuses on addressing these challenges pertinent to existing cellular communication and the $5 G$ feasible solutions.

\section{Vehicle-to-Thing in $5 G$}

\subsection{System Architecture}

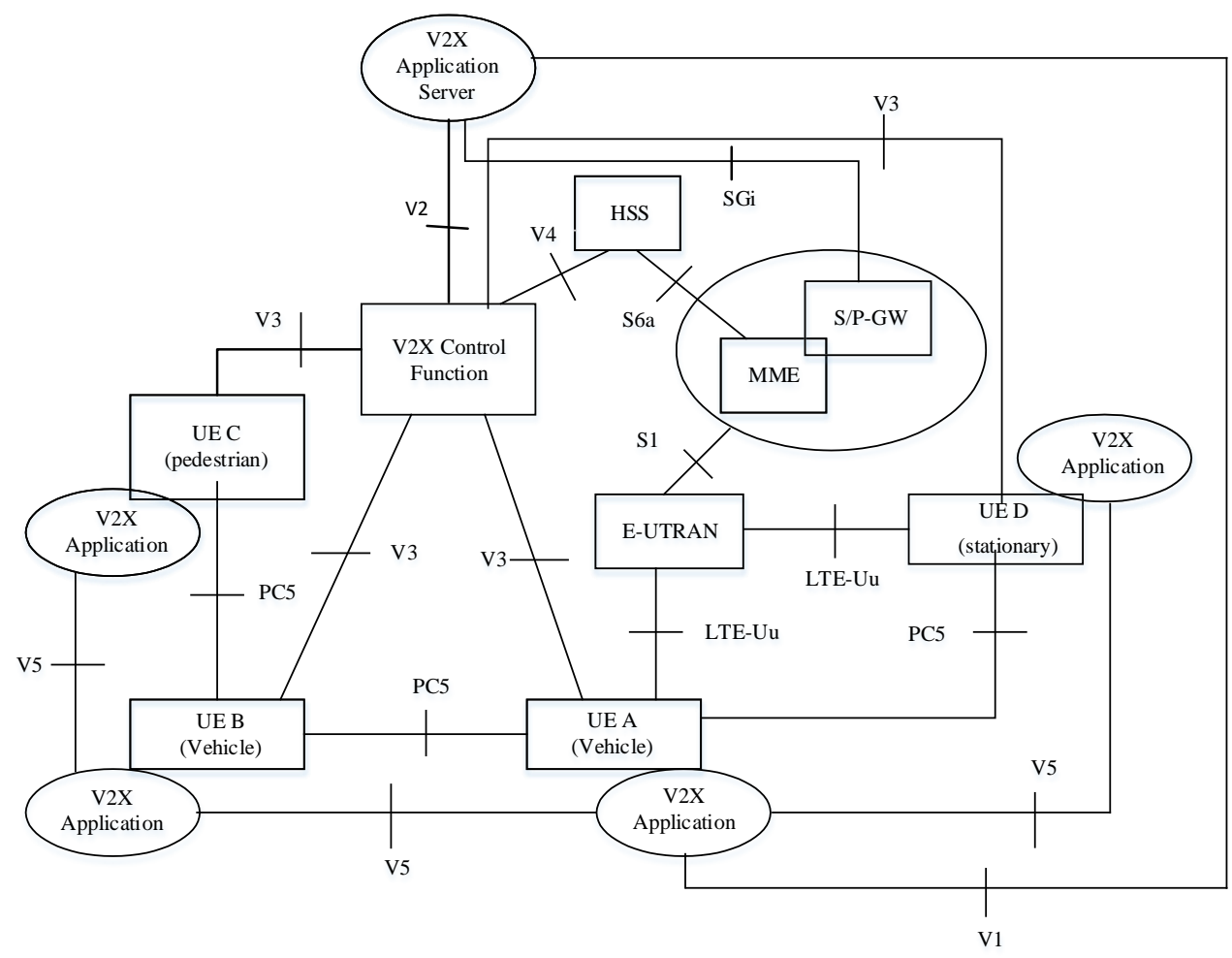

Figure 1 The architecture of LTE-V2X 
V2X services can be provided by PC5 interface and/or LTE-Uu interface. Support of V2X services by PC5 interface is provided by V2X sidelink communication, which is a mode of communication whereby UEs can communicate with each other directly over the PC5 interface. LTE-Uu interface and PC5 interface is used separately when the UE is served by E-UTRAN and when the UE is outside of E-UTRA coverage. Figure 1 is a reference model of the architecture of LTE-V2X [9].

Compared to the architecture of LTE D2D [10], the architecture of LTE-V2X adds V2X Control Function. V2X Control Function is logical entity of network for V2X, provides the UE with necessary parameters to allow UE to use V2X, and is discovered through the interaction with Domain Name Server (DNS) function. Assume that UE implement V2X with PC5 reference point, UE obtain authorization from the V2X Control Function of Home Public Land Mobile Network (HPLMN), afterward UE receives authorization message and parameter (e.g. authorization policy, radio parameter (i.e. carrier frequency)). Suppose that UE uses LTE-Uu interface point for V2X communication, the V2X Control Function should configure the following information and optionally provide information to the UE: PLMN which the UE is authorized to use MBMS with V2X, V2X Application Server address information, the mapping of V2X services.

\subsubsection{Authorization for LTE-V2X}

The two basic principles of service authorization for V2X communication over PC5. First, UE gets authorization from V2X Control Function in HPLMN. Second, V2X Control Function merges authorization information from home and serving PLMN. And authorization policy, radio parameters and policy are provisioned by the V2X Control Function to UE. Authorization policy is whether the UE is authorized to perform V2X communication over PC5 reference point. Radio parameters (e.g. frequency bands) is related to Geographical Area(s). Policy includes the mapping of Destination Layer-2 ID(s) and the V2X services, includes the mapping of ProSe Per-Pacet Priority and packet delay budget, includes the mapping of service types and V2X frequencies, include the list of V2X service types with Geographical Area(s).

The following information optionally provisioned to the UE for V2X communication over LTEUu reference point: first, the PLMN is authorized to use MBMS based V2X communication; second, V2X Application Server address information; third, mapping of V2X services to V2X Application Server address and V2X USD for MBMS. 


\subsubsection{V2X message transmission/reception}
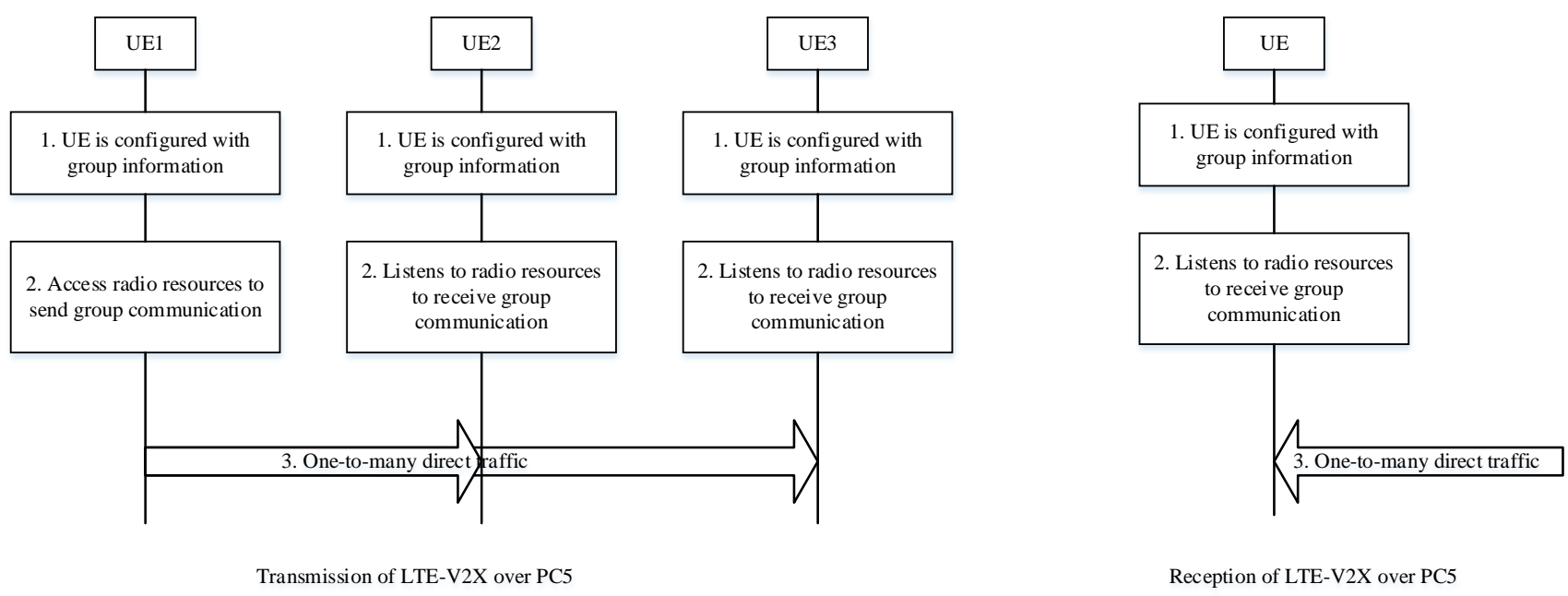

Reception of LTE-V2X over PC5

Figure 2 LTE-V2X communication over PC5 reference point

The UE is authorized to use LTE-V2X communication, it can transmit or receive messages. Oneto-many ProSe Direct Communication is applied to LTE-V2X communication over PC5 reference point[11][12]. As the descried by Figure 2, UE perform the transmission of LTE-V2X communication over PC5 reference point, UE receives the necessary group context (i.e. ProSe Layer-2 Group ID, ProSe Group IP multicast address) and radio resource parameter. UE finds the appropriate radio resource to one-to-many communication, then UE sends the IP data to the IP multicast address. When UE perform the reception of LTE-V2X communication over PC5 reference point, the UE obtains the necessary group context ( ProSe Layer-2 Group ID, Group IP multicast address) to receive IP-layer transport of data, and the radio resource related parameters used for communication. The UE listens to allocate radio resources to receive the information of communication. The receiving UE filters out the received frames based on the ProSe Layer-2 Group ID contained in the Destination Layer-2 ID and if it matches one of the configured Group IDs, it delivers the enclosed packet to the upper layers.

UE performing LTE-V2X communication over LTE-Uu reference point need two procedures: first, V2X Application Server discovery using broadcast; second, procedure for V2X communication with MBMS. The procedure of V2X Application Server discovery using broadcast is defined in Figure 3. UE attaches to the serving PLMN, and receives local Service Information (including the Fully Qualified Domain Name (FQDN) of server, V2X User Service Description (USD)) from V2X Control Function. The UE obtains the IP address of V2X Application Server using DNS protocol with FQDN. And UE may connect with V2X Application Server for service. The procedure of V2X communication with Multimedia Broadcast Multicast Services (MBMS) is defined in Figure 4. UE provides its geographic location or Cell ID information over V1 inference point. The V2X Application Server uses this information to map the target broadcast area and/or Cell ID information, then deliver the information to the BM-SC. The BM-SC derives the MBMS Service Area and the SAI list for the availability information depending on the geographic location information from V2X Application Server. From such procedure, the V2X Application Server knows which MBMS session is serving a geographical area. Hence, V2X Application Server forwards a V2X message to the appropriate MBMS session. 


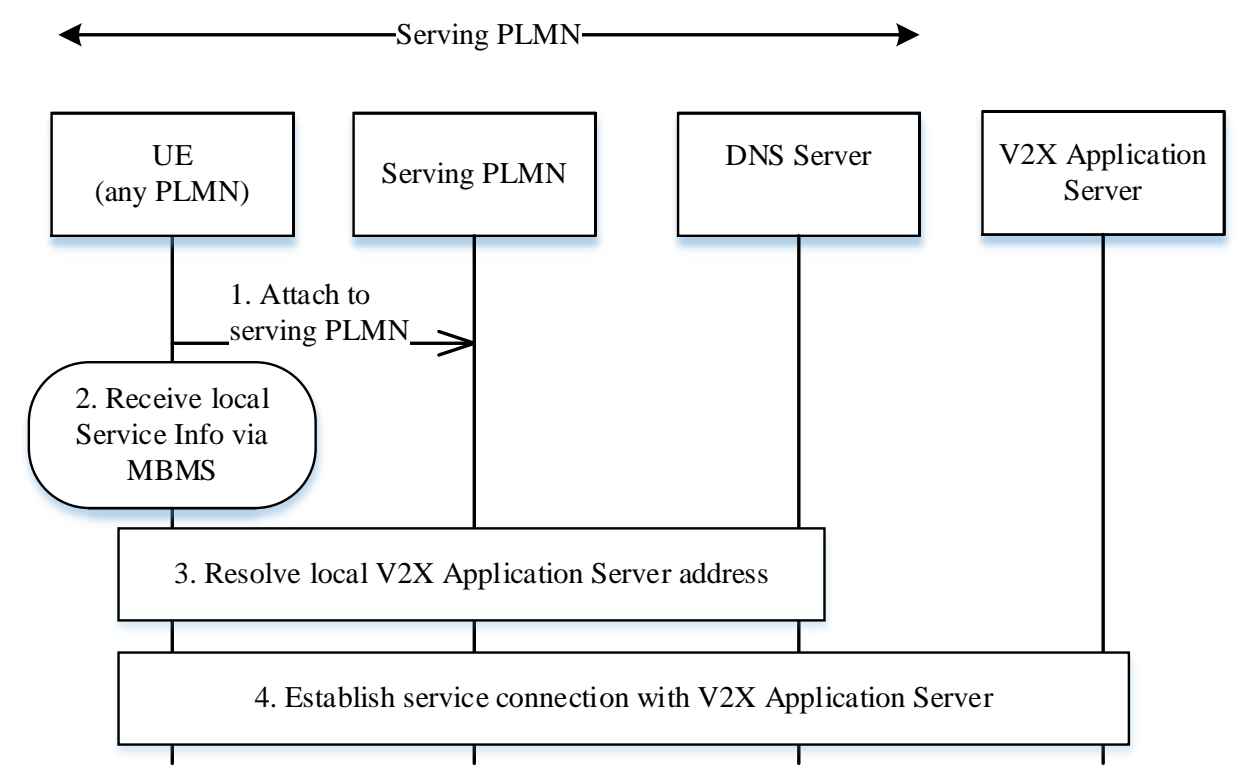

Figure 3. V2X Application Server discovery

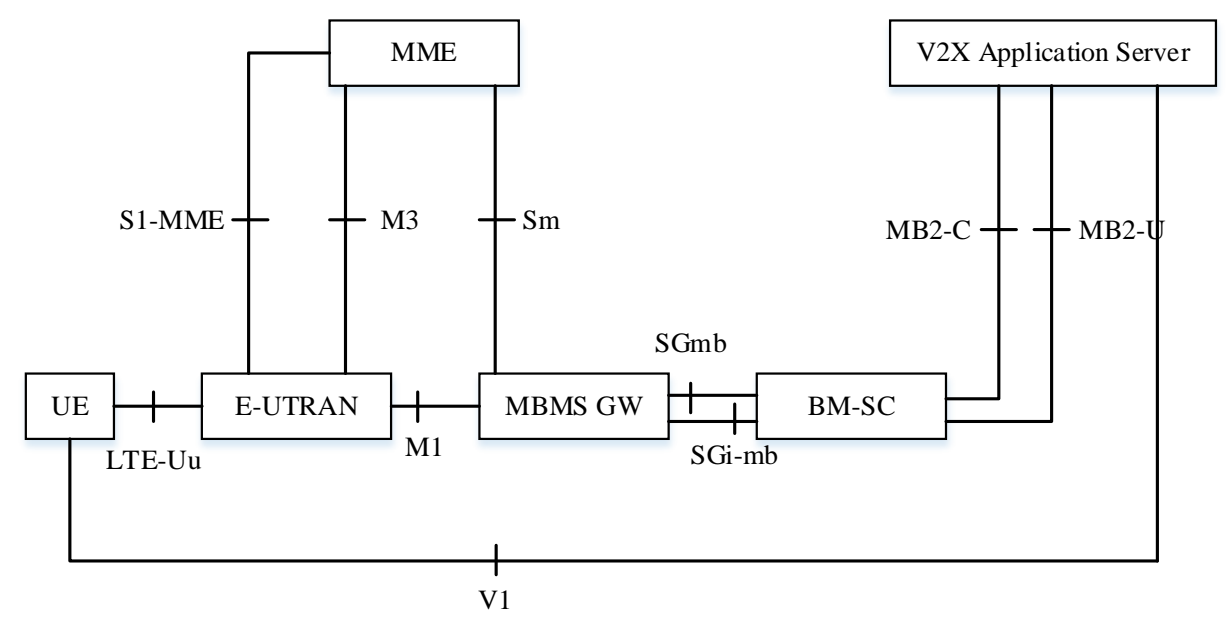

Figure 4. V2X communication with MBMS

\subsection{LTE-V2X Sidelink Communication}

In LTE-V2X sidelink communication, 3GPP inherits their idea of D2D resource allocation and improves it, also add new transmission modes called Mode 3 and Mode 4.To enhance the channel estimation accuracy for high speed vehicles, the number of Demodulation Reference Signal (DMRS) subframes has been increased from two to three per resource block (RB) for the Physical Sidelink Broadcast Channel (PSBCH) and to four for the Physical Sidelink Control Channel (PSCCH) and Physical Sidelink Shared Channel (PSSCH) in order to account for the high Doppler spread. In mode 3, UE requests transmission resources from the eNB. The eNB schedules transmission resources for transmission of sidelink control information (SCI) and data. In mode 4, vehicles autonomously selects resources from resource pools and performs transport format selection to transmit sidelink control information and data.

The Sidelink Control Information (SCI) Format 1 was introduced in 3GPP Release 14 (D2D uses SCI Format 0). It is transmitted over the Physical Sidelink Control Channel (PSCCH), which carries the information related to the transmission of data over the Physical Sidelink Shared Channel 
(PSSCH). The SCI Format 1 informs the receiving vehicular UEs about the resource reservation interval, frequency location of initial transmission and retransmission (up to one retransmission is supported in Mode 4), time gap between initial transmission and retransmission (i.e. SFgap), and modulation and coding scheme (MCS) used to modulate the data transmitted over the PSSCH. The SCI is transmitted in the PSCCH and the data is transmitted in the PSSCH. The UE wants to know that the content in data must decode the information in the SCI first. The SCI includes information on the modulation and coding scheme (MCS).

LTE-V2X defines a series of RBs as a resource pool in the same subframe. Resource locations occupied by the resource pool can be determined by parameters (i.e. start-RB, sizeSubchannel, and numSubchannel). In this resource pool, include the SCI and the corresponding data. The SCI is transmitted in the PSCCH and the data is transmitted in the PSSCH. The UE wants to know that the content in data must decode the information in the SCI first. The SCI includes information on the modulation and coding scheme (MCS), the RBs it uses, and the resource reservation interval for semi-persistent scheduling (SPS). LTE-V2X defines two subchannelization schemes: adjacent and non-adjacent. The adjacent scheme: SCI and data are transmitted in adjacent RBs, each SCI occupies the first two RBs of the first subchannel, the RBs of data follows RBs of SCI. Nonadjacent scheme: The RBs are divided into pools. One pool is dedicated to transmit only SCIs, and the SCIs occupy two RBs. The second pool is reserved to transmit TBs and is divided into subchannels.

\subsubsection{Centralized Allocation (mode 3)}

A UE in RRC_IDLE that is configured by upper layer to transmit V2X sidelink communication, and receives the SystemInformationBlockType21 (SIB21) defined in Figure 5 which is broadcast by Cell. When the receiving SIB21 does not include the resources for transmission (i.e. $v 2 x$ CommTxPoolNormal, p2x-CommTxPoolNormal), an RRC connection is initiated. When the UE in RRC_CONNECT changes the interest (such as handover), it will transmit the SidelinkUEInformation message, and the EUTRAN modify the RRC connection, it initiates the RRC connection reconfiguration procedure. The above procedure is defined in Figure 6.

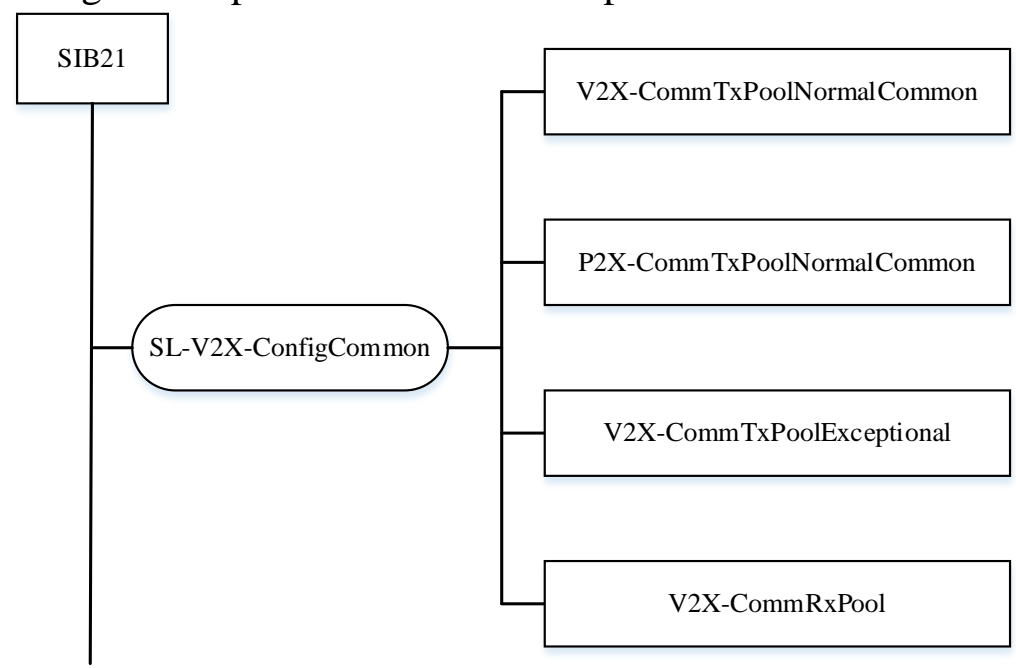

Figure 5. SIB21 


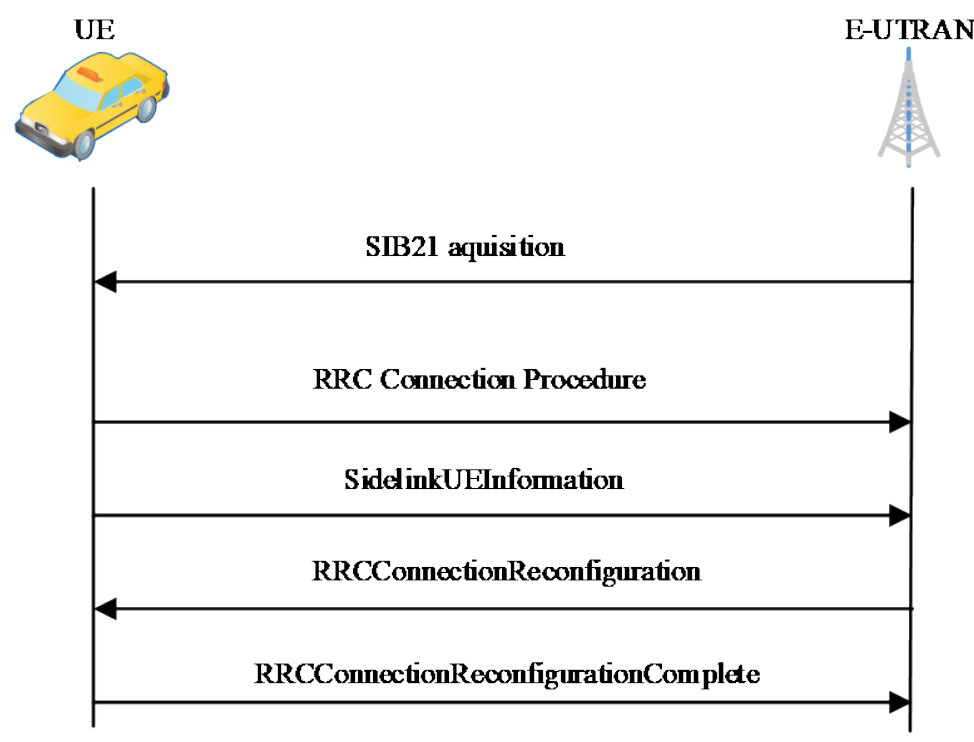

Figure 6. Resource Allocation of TM3

\subsubsection{Distributed Allocation (mode 4)}

Vehicle automatically select resources for V2X sidelink communication. When in the coverage, network supports configuration that includes resource pool for V2X, synchronization reference, the number of sub-channels in a subframe, and the number of RB in each subchannel. And UE is out of coverage, vehicle uses the pre-configuration.

The procedure of resource selection, defined in Figure 7, as following: first, The upper layer requests in subframe $\mathrm{n}$. Contiguous sub-channels included in the corresponding PSSCH resource pool within the time interval $\left[\mathrm{n}+\mathrm{T}_{1}, \mathrm{n}+\mathrm{T}_{2}\right]$ corresponds to one candidate single-subframe resource (CRS). The time interval is called selection window; second, vehicle senses the 1000 subframs before the subfame $n$, excludes the used resource by receiving SCI and PSSCH-RSRP; finally, vehicle report the candidate single-subframe resource to higher layers.

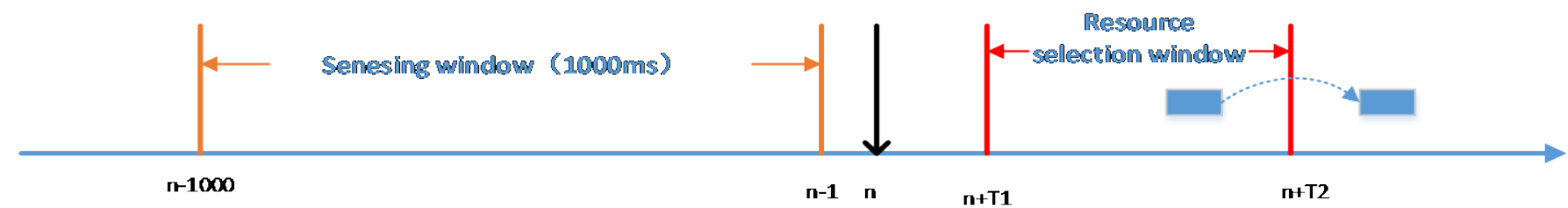

Figure 7. Resource (re)selection for mode 4

\subsection{The New Feature of LTE-V2X}

The latency of traditional LTE cannot match the requirement of V2X, e.g. Maximum latency between $3 \mathrm{~ms}$ and $10 \mathrm{~ms}$. In order to satisfy the nearly demanding requirements, we proposed some feasible solutions. Radio resource pool sharing improve resource utilization, latency reduction to achieve the latency requirement.

\subsubsection{Radio resource pool sharing between UEs}

In release 14, resource pools used by mode 3 and mode 4 are independent, and do not overlap. 
The advantage of dedicated resource pool lies in the collision avoidance between mode 3 UE and mode 4 UE since their resources come from different resource pools. But the dedicated resource pool may cause resource pools to be unbalanced, one resource pool is severe overloaded, and the other resource is underutilized. Therefore resource pool sharing can be considered, with the performance of the original system cannot be significantly reduced and the system complexity cannot be significantly higher. There are three scenarios: release15-mode4-UE and release14mode3-UE, release15-mode3-UE and release14-mode4-UE, release15-mode3-UE and release15mode4-UE. However, for the mixed mode-3 and mode-4 scenarios, the open question is how to avoid scheduling of mode- 3 transmissions on resources occupied by mode-4 UEs or avoid using mode-3 resources by mode-4 UEs.

Sharing of release15-mode4-UE and release14-mode3-UE. The resource reservation field of release14-mode3-UE is set to "0000", so release15-mode4-UE cannot forecast a release14-mode3UE's transmission based on decoded release14-mode3-UE SCI indication in the sharing resource pool. But the release15-mode4-UE can use the reservation bits of the SCI to identify the release14UE, and identifies the transmission mode by the pool configuration. For Rel-14 UEs, the resource pool is mode-specific, thus release15-mode4-UE knows which mode the release14-UEs are using. Then release15-mode4-UE automatically excludes the resource of release14-mode3-UE without comparing the RSRP with the threshold.

Sharing of release15-mode3-UE and release15-mode4-UE. In release 14, mode3-UE does not perform the step of sensing resources. The process of sensing resources is applied to achieve resource pool sharing. Release15-mode3-UE senses the resources, and then reports to the eNB. The eNB allocates resources to the R15-mode3-UE according to the content of the report. But this bring two problems: overhead, release15-mode3-UE perform sensing and report the result of sensing; latency, in the sending sense results may also occur collision, interaction time (such as SR / BSR, DCI) between UE with eNB may be larger, which may increase latency. To solve the above two problems, the following improvements can be consideration. For overhead, the eNB sends signaling to let the mode3-UE perform sensing, otherwise the UE does not perform sensing; after the UE senses the resource pool, only the lastest candidate resource is reported, not all available candidate resources. For latency, reporting and data transmission are performed independently. Reporting may be performed periodically or in half cycles, the eNB may schedule resources for the UE according to the latest reporting. For release15-mode4-UE, using SPS mechanism to avoid collision.

Sharing of release15-mode3-UE and release14-mode4-UE. Since the release14-mode4-UE cannot recognize the any new introduced indication in release 15 , so we can consider to enhance the release15-mode3-UE. In release 14, mode4-UE sense the resources in pool, and specify the resource reservation field and priority indicator in SCI format 1 to indicate the occupied/reserved resource and its priority level, seeing the Table 1 . After sensing, release14-mode4-UE can avoid selecting the resource which is occupied by the other UE with higher priority. By the way, we propose to use the resource reservation field in SCI 1 format for release15-mode3-UE. eNB can indicates the resource reservation field for release15-mode3-UE to reserve resources for dynamic transmission. From the system's perspective, eNB can set the reservation resource for multiple UE, even the eNB can set the higher priority for mode3-UE, and then the mode3-UE can avoid selecting the reservation resources. 
Table 1 Determination of the Resource reservation field in SCI format 1

\begin{tabular}{|c|c|c|}
\hline $\begin{array}{c}\text { Resource } \\
\text { reservation field } \\
\text { in SCI format } 1 \\
\end{array}$ & $\begin{array}{c}\text { Indicated value } \\
\qquad X\end{array}$ & Condition \\
\hline $\begin{array}{c}\text { '0001', '0010', ..., } \\
\text { '1010' }\end{array}$ & $\begin{array}{l}\text { Decimal } \\
\text { equivalent of the } \\
\text { field }\end{array}$ & $\begin{array}{l}\text { The higher layer decides to keep the resource for the } \\
\text { transmission of the next transport block and the value } X \\
\text { meets } 1 \leq X \leq 10 \text {. }\end{array}$ \\
\hline '1011' & 0.5 & $\begin{array}{l}\text { The higher layer decides to keep the resource for the } \\
\text { transmission of the next transport block and the value } X \text { is } \\
0.5 \text {. }\end{array}$ \\
\hline '1100' & 0.2 & $\begin{array}{l}\text { The higher layer decides to keep the resource for the } \\
\text { transmission of the next transport block and the value } X \text { is } \\
0.2 \text {. }\end{array}$ \\
\hline '0000' & 0 & $\begin{array}{l}\text { The higher layer decides not to keep the resource for the } \\
\text { transmission of the next transport block. }\end{array}$ \\
\hline $\begin{array}{l}\text { '1101', '1110', } \\
\text { '1111' }\end{array}$ & Reserved & \\
\hline
\end{tabular}

With the analysis of three coexistence scenarios, we give the following suggestion:

(1) Using one bit of the reservation field of SCI format 1 to indicate the mode of UE, e.g. ' 1 ' represents mode 4 and ' 0 ' represents mode 3.

(2) Using resource reservation field of SCI format 1 for UE of mode 3, by this way the UE of mode4 of release 15 can decode the SCI information of release15-mode3-UE.

(3) Performing the sensing process for UE of mode 3, UE of mode3 of release 15 send the report to the eNB.

How to configure the resource pool sharing is also need to be solved. The pool configuration can be raised: two dedicated pool configure to mode 3 and mode 4, a sharing pool for both mode 3 and mode 4, liking in the left of Figure 8, and UE priority select the private dedicated pool. When a little of UE in cell, UE use the dedicated pool. And when there are large number of UE, some of they use the sharing pool. And the configuration has another function, when there is only one mode of crowded UE as the right of Figure 8, the mode3-UE can select the resource without sensing, and it will not cause collision.

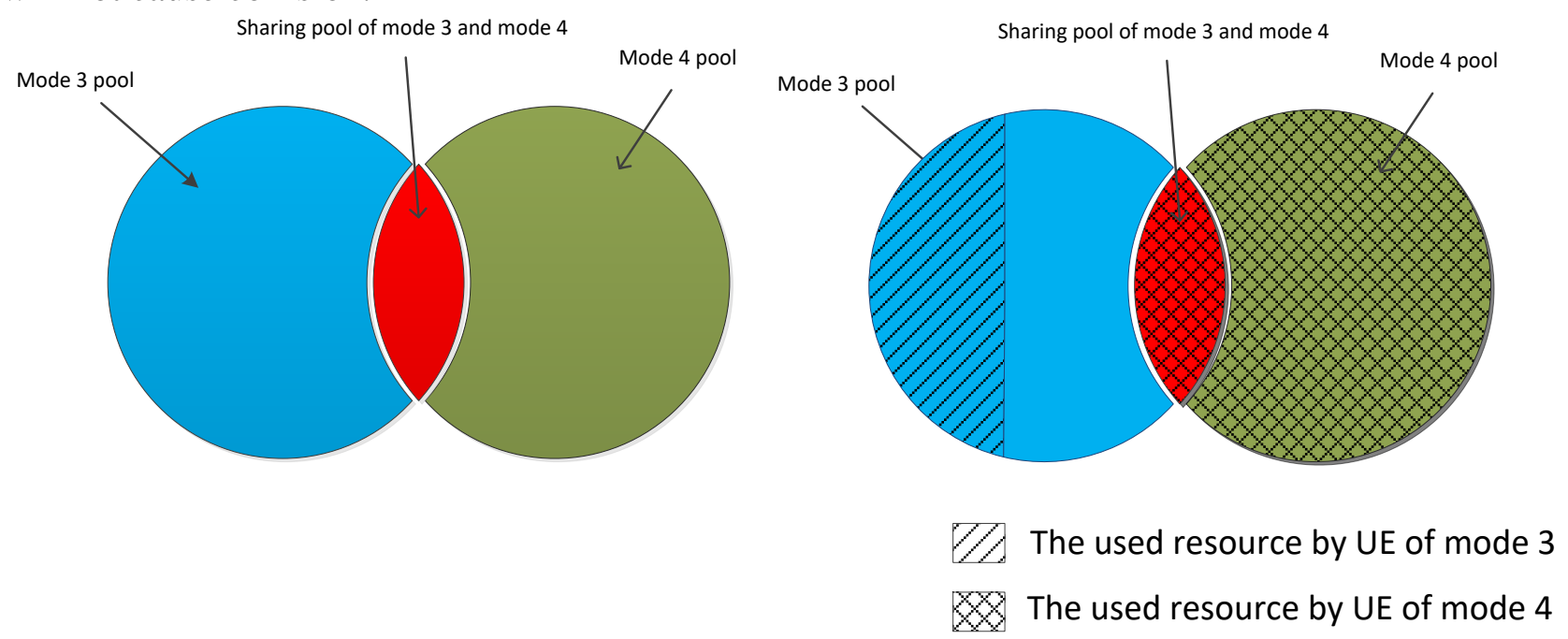

Figure 8. Pool configuration 
The size of sharing pool is also important. The smaller size, the lower resource utilization and the smaller collision. Thereby, it is tradeoff between resource utilization and collision. Simulation results show that the size of sharing pool is larger than $58 \%$ of dedicate resource of release 14 causes the raising collision. So we have an agreement that the size of sharing pool cannot exceed the 58 percent of dedicate resource in release 14, the result of its simulation is defined in Figure 9.

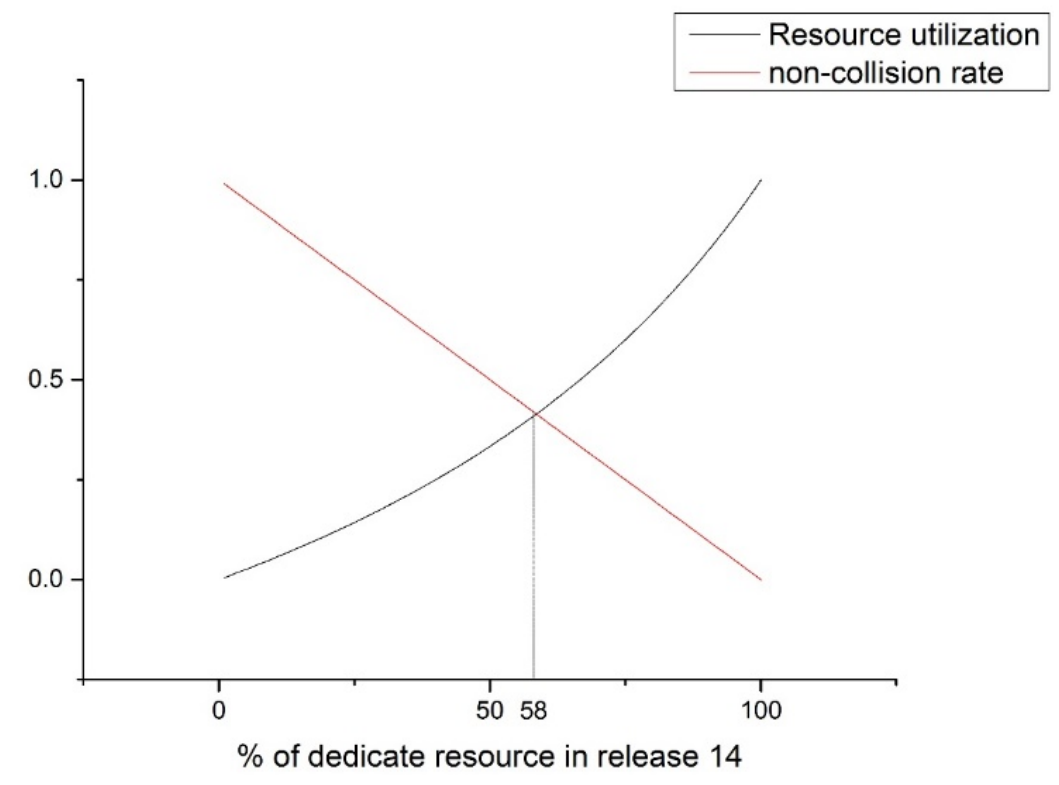

Figure 9. The simulation of resource utilization and non-collision rate

So with the resource configuration, the process of resource selection can be described as following: when the resource of dedicated pool is sufficient, UE can independently select resource as the process of release 14 . And the resource of dedicated pool is poor, and UE select the resource from the sharing pool, the release15-mode4-UE decode the receiving SCI, exclude the resource used by release15-mode3-UE depending on the reservation resource field of SCI. When the release15-mode3-UE select the resource from the sharing resource pool, the eNB sends signal to the UE to sense the resource sharing pool, the UE sense and report sensing result to the eNB to assist eNB scheduling. As the Figure 10, When the resource of mode3-pool is exhausted, and UE2 uses the resource of sharing pool, eNB sends the sensing signaling to UE2. The UE2 senses the resources by decoding the SCI from others, find the resource R8 is clear. And UE2 marks the R8 as candidate resource, and send the result (i.e. CRS) to the eNB. 

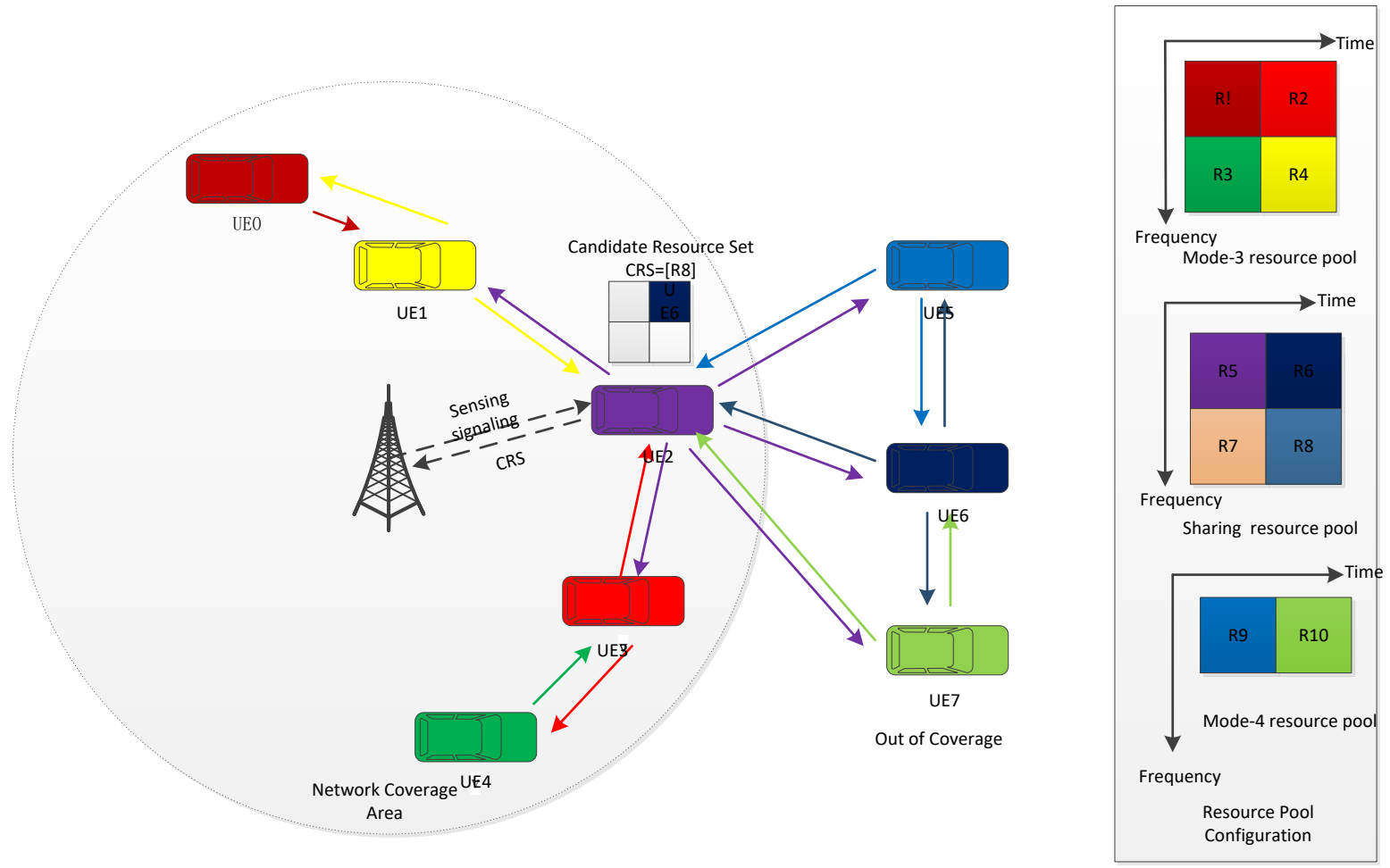

Figure 10. Resource Scheduling

\subsubsection{Latency Reduction}

Autopilot, remote driving, and cloud driving are future trends, and supporting these applications involves a key technical parameter: low latency. In TR22.886, it is pointed that a fleet with a speed of $100 \mathrm{~km} / \mathrm{h}$ has an interval of 2 meters per vehicle, a message sending frequency of 40 messages per second, a message size of 300-400 bytes, and end-to-end delay support 25ms; when the vehicle is more and more dense and the interval between vehicles is $1 \mathrm{~m}$, the message sending frequency is 100 messages per second, and the message size is 50-1200 bytes, and the end-to-end delay at lease support $10 \mathrm{~ms}$. In the scenarios of the TR22.186, the requirement of latency ranges from 3ms to $100 \mathrm{~ms}$, and the typical range is $10 \mathrm{~ms}$ to $20 \mathrm{~ms}$. Release 14 can only support the minimum latency of 20ms. Obviously, it cannot reach the latency requirement of release 15. Inherence needs to reduce latency.

\subsubsection{Latency Reduction in Mode 4}

At this stage, the main topics for reducing latency of mode4 at recent meetings were to reduce the width of the resource selection window, and more specifically to reduce the final time point of the selection window. For Rel-14 V2X, packets arrive at MAC TX buffer, MAC requests Physical layer for candidate resource, and then randomly selects resources within the candidate resource.

In mode 4, using SPS resource scheduling as Figure 9 . If the packets arrive at time $\mathrm{n}$, the UE listens to the resource pool in the sensing window [n-1000 n-1], and then selects the transmission resource in the resource (re)selection window [n+T1 n+T2] according to the sensing result. The maximum latency using the SPS resource scheduling method is determined by T2 $(20<\mathrm{T} 2<100)$. To meet the delay requirement of release 15, the value of T2 should be reduced. In terms of the fundamental limits that $1 \mathrm{~ms}$ subframe physical structure and receiver implementation (e.g. processing delays up to $4 \mathrm{~ms}$ ), therefore the L1 latency reduction is bounded by at least $5 \mathrm{~ms}$ in practical implementations, and we have a proposal that The minimum (pre-)configurable T2 is 5ms. 
By the way, the selection window size is shorten, and the number of the candidate resource may not meet the requirement of $20 \%$ of all the resource in selection window. But the physical layer still reports at least $20 \%$ candidate resource of $A$ by increasing the threshold. It will reduce the range of sidelink transmission and increase collision probability. So the decrement percentage of $A$ and the increment threshold is insufficient, a solution to address the issue is to extend the resource selection window in the frequency domain. UE can consider single-subframe resources within [n+T1,n+T2] on multiple carriers liking in the Figure 11.

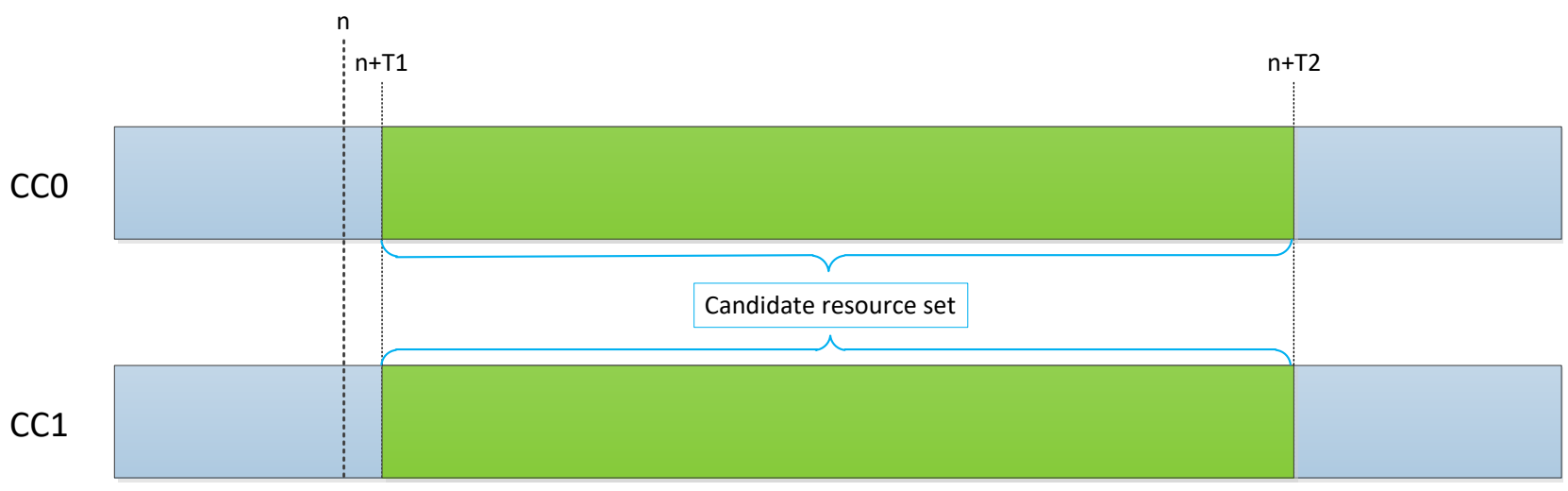

Figure 11 resource selction window [n+T1,n+T2] on multiple carriers

\subsubsection{Latency reduction in Mode 3}

With mode3, if UE has no sidelink grant when V2X packet arrives, the latency of mode 3 may be up to 19.5ms. And the latency consists of the following, descried as Figure 12 and Table 2.

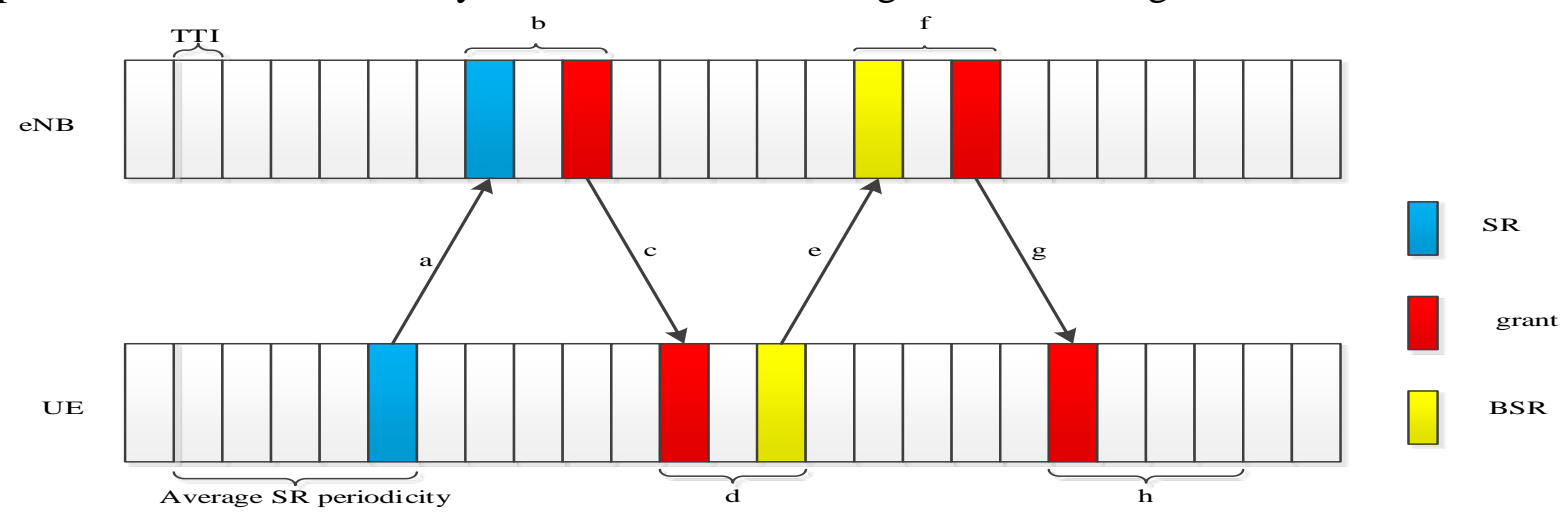

Figure 12. The Content of Latency over Mode 3 
Table 2. Normal SR/BSR Latency component

\begin{tabular}{|c|c|c|}
\hline Component & Description & Time \\
\hline & $\begin{array}{l}\text { Average waiting time for PUCCH } \\
\text { (5 TTI SR period) }\end{array}$ & $2.5 \mathrm{TTI}$ \\
\hline $\mathrm{a}$ & $\begin{array}{l}\text { UE sends Scheduling Request } \\
\text { (SR) on PUCCH }\end{array}$ & $1 \mathrm{TTI}$ \\
\hline $\mathrm{b}$ & $\begin{array}{c}\text { eNB decodes Scheduling Request } \\
\text { and generates the Scheduling } \\
\text { Grant }\end{array}$ & 3 TTI \\
\hline C & Transmission of Scheduling Grant & $1 \mathrm{TTI}$ \\
\hline $\mathrm{d}$ & $\begin{array}{c}\text { UE Processing Delay (decoding } \\
\text { of grant }+ \text { L1 encoding of UL } \\
\text { data) }\end{array}$ & 3 TTI \\
\hline e & UE sends BSR & $1 \mathrm{TTI}$ \\
\hline $\mathrm{f}$ & $\begin{array}{l}\text { eNB decodes BSR and generates } \\
\text { scheduling grant }\end{array}$ & $3 \mathrm{TTI}$ \\
\hline g & Transmission of scheduling grant & $1 \mathrm{TTI}$ \\
\hline h & $\begin{array}{c}\text { UE processing delay and } \\
\text { transmission (decoding } \\
\text { scheduling grant }+\mathrm{L} 1 \text { encoding of } \\
\text { data) }\end{array}$ & $4 \mathrm{TTI}$ \\
\hline Total & & $2.5=19.5 \mathrm{TTI}$ \\
\hline
\end{tabular}

Analysis of the contents of latency over mode 3, We have the following findings: the time of sending information between UE and eNB is fixed; the time of UE or eNB decoding information are proportional to the TB size, reducing TB size is useful for low latency, the requirement of V2X scenarios, the size of payload of message is 50-12000 bytes, so shrinking the size of TB is unwise to V2X. And consideration of compatibility, the procedure cannot be simplified. Thereby, low latency implementation can be achieved by shorten TTI. With the decrease of TTI length, processing speed of both UE and eNB sides will be improved and the waiting period of SR can be decrease, and also shorten TTI cannot decrease the throughput of system, the result of simulation as Figure 13.

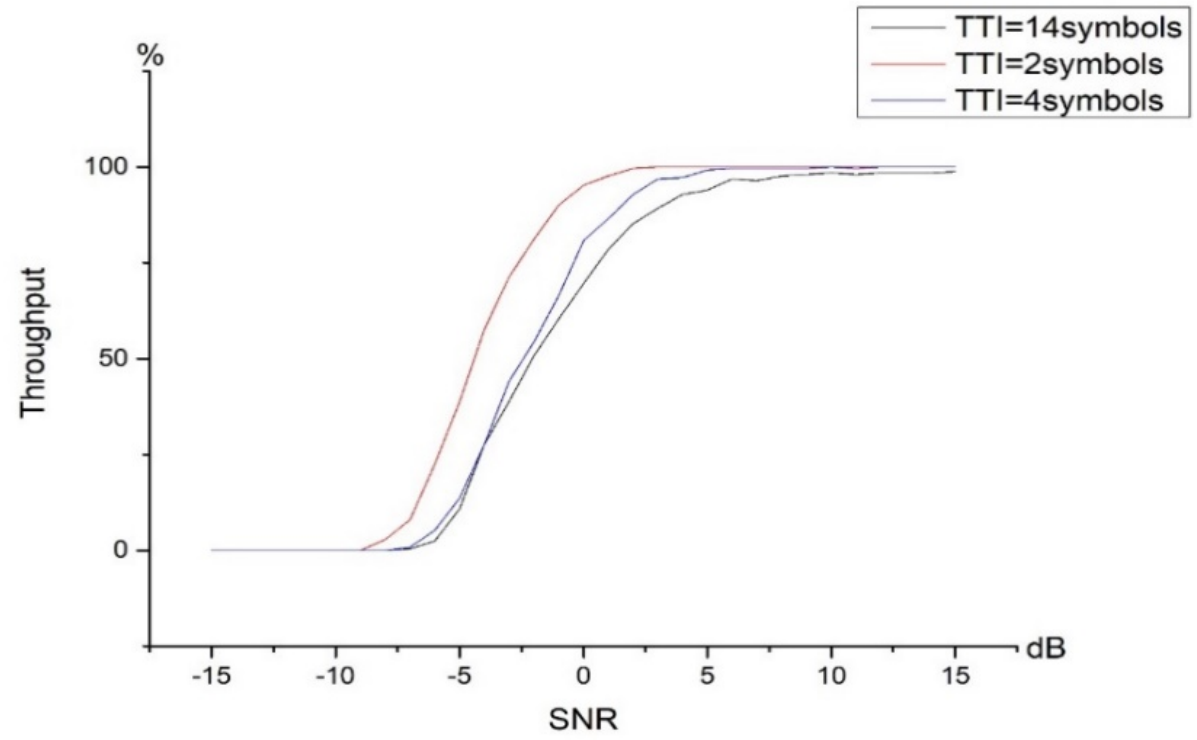

Figure 13. Throughput of different TTI length

In the current version of LTE, the length of TTI is 1ms, is equal to the duration of a subframe which consists of two slots containing 14 OFDM symbols. Consideration of DMRS symbol, the 
TTI length must lager than 1 symbol. So the TTI length can be from $1 \mathrm{~ms}$ to one slot, i.e. $0.5 \mathrm{~ms}$, or to two symbols, i.e. $0.14 \mathrm{~ms}$. Table 4 illustrates latency can be decreased by shortening TTI length.

Table 1. Latency analysis depending on shorten TTI length

\begin{tabular}{ccccccccccc}
\hline $\begin{array}{c}\text { TTI } \\
\text { length } \\
\text { (ms) }\end{array}$ & $\begin{array}{c}\text { Waiting } \\
\text { for SR } \\
\text { (ms) }\end{array}$ & $\begin{array}{c}\mathbf{a} \\
(\mathbf{m s})\end{array}$ & $\begin{array}{c}\mathbf{b} \\
\mathbf{( m s})\end{array}$ & $\begin{array}{c}\mathbf{c} \\
\mathbf{( m s})\end{array}$ & $\begin{array}{c}\mathbf{d} \\
\mathbf{( m s})\end{array}$ & $\begin{array}{c}\mathbf{e} \\
\mathbf{( m s )}\end{array}$ & $\begin{array}{c}\mathbf{f} \\
\mathbf{( m s})\end{array}$ & $\begin{array}{c}\mathbf{g} \\
(\mathbf{m s})\end{array}$ & $\begin{array}{c}\mathbf{h} \\
(\mathbf{m s})\end{array}$ & $\begin{array}{c}\text { Total } \\
(\mathbf{m s})\end{array}$ \\
\hline 1 & 2.5 & 1 & 3 & 1 & 3 & 1 & 3 & 1 & 4 & 19.5 \\
0.5 & 1.25 & 0.5 & 1.5 & 0.5 & 1.5 & 0.5 & 1.5 & 0.5 & 2 & 9.75 \\
0.14 & 0.36 & 0.14 & 0.42 & 0.14 & 0.42 & 0.14 & 0.42 & 0.14 & 0.56 & 2.74 \\
0.28 & 0.72 & 0.28 & 0.94 & 0.28 & 0.94 & 0.28 & 0.94 & 0.28 & 1.12 & 5.48 \\
\hline
\end{tabular}

The existence of DMRS, the lower the TTI length, the lower the resource efficiency. Two symbols of TTI length has only 50\% resource utilization, four symbols (1 symbol for DMRS ) of TTI length has 75\% resource utilization, and current design in 3GPP that 14 symbols per 1TTI (4 symbols for DMRS) has 71.4\%. Therefore, the TTI length must be a compromise between latency and resource utilization. According to the simulation in Figure 13 and the resource utilization, we suggest using 4 symbols per TTI.

\subsubsection{Carrier Aggregation}

One of the characteristics of $5 \mathrm{G}$ is ultrahigh rate, and carrier aggregation can increase the peak data rate. The content of Downlink Control Information format 5A includes carrier indictor that the field occupies 3 bits, so the carrier aggregation is up to 8 carriers.

When a MAC PDU is arrived, the procedure of resource selection as following step. Step 1: the high layer supports the set of candidate carriers for transmission; Step 2: MAC PDUs is arrived, the UE selection the carrier for actual transmission; Step3: UE select resource according to sensing. Once the UE gets enough resources, it sends the MAC PDUs. There is discussion on carrier selection and resource selection, poor carrier selection solution will cause unbalanced load between the carriers. Our opinion is that UE monitor the load of carriers and select the carrier according to the Channel Busy Ratio (CBR), this solution is more accurate and the measurement is in the granularity of the resource pool, which can reflect the overall load of each carrier. Source selection solution is also consideration. When UE transmits/receives packets on a carrier, it cannot receive/transmit packets on the same/adjacent carrier, the half-duplex lead to the all subframes cannot be included in the sensing window in a carrier. Consideration the half-duplex, component carrier cannot independently select resource. The component carrier should synchronize. In the LTE, there are three synchronization reference: eNB, GNSS, UE. Consideration the frequency shift, a single synchronization reference is used for all aggregated carriers. And there would be timing misalignment among different carriers, timing on all transmission carriers is aligned.

\section{Acknowledgments}

The paper gives exhaustive explanation of vehicular communication based on cellular communication and vehicle getting the service from cellular communication over PC5 or Uu reference interface. To meet the new features in 5G V2X that extremely low latency, high-efficient communication and ultrahigh rate, the possible 5G solution are analyzed. Shorten TTI length in mode 3 and shrink the resource (re)selection window in mode 4 to enable latency requirement, raising a resource sharing mechanism to increase the resource utilization, and analysis the problems that carrier aggregation apply in vehicular IoT. 


\section{References}

[1] Shanzhi Chen et al., “LTE-V: A TD-LTE-Based V2X Solution for Future Vehicular Network”, IEEE Internet of Things Journal, vol. 3, no. 6, Dec. 2016, pp.997-1005.

[2] E. Hamida, H. Noura, and W. Znaidi, "Security of Cooperative Intelligent Transport Systems: Standards, Threats Analysis and Cryptographic Countermeasures,” Electronics, vol. 4, no. 3, pp. 380-6423, 2015.

[3] I. Ivanov, et al., "Cyber Security Standards and Issues in V2X Communications for Internet of Vehicles”, in Cybersecurity of IoT conference, London, March 2018, pp.1-6.

[4] Konstantinos Manolakis, et al., “Time Synchronization for Multi-Link D2D/V2X Communication”, IEEE Vehicular Technology Conference, Montreal, Mar. 2016.

[5] H. Tang, Z. Ding, and B. C. Levy, "Enabling D2D Communications Through Neighbor Discovery in LTE Cellular Networks,” IEEE Transactions on Signal Processing, vol. 62, no.19, pp. 5157-5170, 2014.

[6] Antonio Celesti et al., "An IoT Cloud System for Traffic Monitoring and Vehicular Accidents Prevention Based on Mobile Sensor Data Processing”, IEEE SENSORS JOURNAL, VOL. 18, NO. 12, JUNE 15, 2018, pp. 4795-4802.

[7] H. Al Najada and I. Mahgoub, "Big vehicular traffic data mining: Towards accident and congestion prevention," in Proc. Int. Wireless Commun. Mobile Comput. Conf. (IWCMC), Sep. 2016, pp. 256-261.

[8] K. Zheng, Q. Zheng, P. Chatzimisios, W. Xiang, and Y. Zhou, "Heterogeneous vehicular networking: A survey on architecture, challenges, and solutions,’’ IEEE Commun. Surveys Tuts, vol. 17, no. 4, pp. 2377-2396, 4th Quart. 2015. [9] 3GPP TS 23.285," Architecture enhancements for V2X services”, Mar. 2018.

[10] 3GPP TS 23.303: "Proximity-based Services (ProSe); Stage 2", Jun. 2017.

[11] 3GPP TR 23.703, Rel. 12,"Study on architecture enhancements to support Proximity-based Services (Prose)”, Feb. 2014.

[12] 3GPP TS 36.213, ”Physical layer procedures”, Dec. 2018. 